\title{
Ulcera Péptica en Pediatría: Experiencia de 10 Años
}

\author{
Dr. John Wenger K. ${ }^{1}$; Dr. Eduardo Hebel W. ${ }^{2}$; Dra. Carmen Gutiérrez T.1 \\ Dr. Ernesto Guiraldes $C^{1}$
}

\section{Peptic Ulcer Disease in Children}

The is a retrospective review of peptic ulcer disease in children from a metropolitan hospital at Santiaga, Chile, over a ten years period (June 1974 - June 1984). The diagnosis was made either by endoscopy or barium meal or both in 31 patients and at surgery in one. In coincidence with the regular use of upper digestive endoscopy, from 1978 , a definite increase in the diagnosis of peptic ulcer was observed. Chronic ulcers were seen in twenty five children and seven had the acute form, the latter being commoner in children less than 6 years of age. The majority of cases with acute ulcers were seen in patients operated on for coartaction of the aorta. Duodenal ulcers predominated over gastric ulcers for both acute and chronic lesions. The overall male to female ratio was $1.8: 1.0$. A positive family history of peptic ulcer was present in $74 \%$ of cases. Most of the patients with chronic ulcers presented with abdominal pain, or gastrointestinal bleeding. Studies of gastric acidity showed higher mean values of basal and maximal acid output in 11 patients with chronic ulers when compared to 12 control children with recurrent abdominal pain in whom peptic uleet disease was ruled out by endoscopy. Twenty seven patients received medical therapy with cimetidine or antiacids. Five clildren required surgical treatment for complications such as massive recurrent hemorthage, perforation or pyloric obstruction.

(Key words: Endoscopy. Peptic Ulcer. Post Operative Stress Ulcers).

Se ha supuesto habitualmente que la úlcera péptica es una entidad de baja prevalencid en nitios $^{1-3}$. Sin embargo en coincidencia con la aplicación de la endoscopía digestiva, este diagnóstico se está hacicndo con frecuencia creciente ${ }^{4-6}$. No ha sido bien precisado si ello se debe a un aumento real de la incidencia, o a mayor eficiencia de la técnica mencionada, en comparación con los exámenes radiológicos convencionales. Por otra parte, es sabido que la incidencia de la úlcera péptica puede variar de un centro a otro dependjendo del entusiasmo con que los clinicos, radiólogos y endoscopistas la buscan.

En esta comunjcación se presenta una experiencia de 10 años en enfermos con úlcera péptica y se analizan sus caracteristicas clínicas, radiológicas y evolutivas.

\section{PACIENTES y METODOS}

La información clínica procede de 32 pacientes en quienes se diagnosticó úlcera gástrica o

1. Unidad de Gastroenterología Infantil, Hospital Luis Calvo Mackenna, Santiago.

2. Departamento de Pediatría, Hospital Regional de Temuco y Universidad de la Froniera. duodenal en el Hospital Luis Calvo Mackenna de Santiago entre Junio de 1974 y Junio de 1984. El diagnóstico se hizo con endoscopía digestiva o estudio radiológico o ambos. En un paciente con abdomen agudo se encontró und ülcera péptica en la laparotomía.

Las úlceras fueron catalogadas como agudas o crónicas. Se definió como úlcera aguda (o securtdaria) la que se presentó durante la evolución de una enfemmedad sistémica grave, que actuó como factor inicial ( septicemia, anoxia, deshidratación grave, elc.). Cuando no se encontró una causa subyacente, la ílcera fue rotulada como crónica (o primaria).

Las endoscopías fueron realizadas con un fibroscopio GIF - P2 usando la técnica descrita en una publicación anterior ${ }^{7}$.

En 11 pacientes con úlcera crónica se hizo sondeo gástrico para medir la acidez basal $y$ máxima. Los resultados fueron comparados con los de un grupo control de 12 niños con dolor abdominal recurrente en quiencs no se encontraron lesiones mediante la endoscopía. La medición de acidez basal y máximu se realizó con el paciente en ayunas: El procedimiento se inició aspirando el contenido gástrico, el que después de medir su volumen, se eliminó (residuo). Luego se recolectó el contenido durante 4 períodos de 
15 minutos cada uno, constituyendo con la suma, el débito basal (BAO). Al término de la segunda recolección se administró Clorprimetón (0,5 mg $\times \mathrm{kg}$, i.m.) y, después de la cuarta, clorhidrato de histamina $(0,024 \mathrm{mg} \mathrm{x} \mathrm{kg}$, con un máximo de $1 \mathrm{mg}$ de dosis total). Luego se continuó aspirando en la forma mencionada durante una hora, para obtener el débito máximo (MAO). La acidez del jugo gástrico se midió por titulación y se expresó en $\mathrm{mEq} \times$ hora $\mathrm{x}$ kg peso.

\section{RESULTADOS}

Se incluyeron 32 pacientes con úlcera péptica, 20 de sexo masculino, con un rango de edad, en el momento del diagnóstico. entre 5 meses y 15 años y un promedio de 7,9 años (Tabla 1 ).

Sólo 5 pacientes ( $16 \%$ ) fueron diagnosticados antes de 1978, año en que sc incorporó la endoscopía digestiva alta a las investigaciones de nuestra Unidad. Los 27 restantes (84\% fueron diagnosticados entre 1978 y 1984 . En 20 cnfermos se practicó endoscopia digestiva como primera etapa de su estuđio. La técnica permitió demostrar una o más úlceras en 19 de ellos. En un caso la endoscopía no fue concluyente y se comprobó radiológicamente una úlcera antral con sindrome de retención gástrića, confirmada durante la intervención quirúrgica. El estudio radiológico se empleó en la investigación jnicial de 20 pacientes, encontrándose úlcera en I8. En dos enfermos cuyas radiografias fucron consideradas normales se demostró, mediante endoscopia, una o más lesiones ulceradas. En 10 niños se emplearon ambas técnicas (endoscopía y radiología) en la primera etapa de estudio. En un caso cl diagnóstico se hizo durante la uperación de una perforación duodenal.
Las úlceras agudas ocurrieron en 7 pacientes, 5 hombres y 2 mujeres; en cuatro la localización fue duodenal exclusiva, en uno gástrica y duodenal, en otro exclusivamente gástrica y, finalmente, en uno esofágica, gástrica y duodenal. El promedio de edad de estos pacientes fue de 1,5 años y en todos habia una afección grave subyacente. Cuatro tenían coartación de la aorta y la úlcera apareció en el período post operatorio inmediato (entre 24 horas y 11 dias) en la forma de melena. Durante el mismo período en que ocurrieron estos 4 casos (Octubre de 1981 a Julio de 1984), 72 pacientes con coartación áórtica fueron sometidos a la operación reparadora, luego la frecuencia de la complicación fue $5,6 \%$. Los otros 3 pacientes con ủlcera aguda tuvieron diarrea aguda con deshidratación $\mathrm{y}$ acidosis; broconeumonia estafilocócica con septicemia $y$ enfermedad poliquística hepato-renal con hipertensión arterial respectivamente.

Las úlceras crónicas ocurrieron en 25 pacien. tes. 15 de sexo masculino. La edad promedio en el momento del diagnóstico fue de 9,7 años. En 23 niños las lesiones se ubicaron en el duodeno, en los dos restantes en el antro gástrico (pre-pilórico). En un caso de úlcera duodenal esta se perforó hacia la vesícula y el páncreas y en 2 pacientes con úlcera pre-pilórica se produjo un sindrome de retención gástrica crónica. Sólo en 19 de los 25 pacientes con úlcera crónica se mencionaron antecelentes de úlcera péptica en familiares. en $14(73,7 \%$ esta habia sido demostrada en uno o más pacientes, en 9 se trataba del padre o la madre, en 5 algún abuelo o tío.

En todos los niños con úlcera aguda el sintoma inicial lue la hemorragia digestiva en ausencia de otros signos. En los enfermos con úlcera crónics, en cambio, el dolor abdominal recurren.

Tabla 1 .

Distribución de las úlceras

\begin{tabular}{|c|c|c|c|c|c|c|c|c|}
\hline \multirow{2}{*}{$\begin{array}{l}\text { Edad } \\
\text { (años) }\end{array}$} & \multirow{2}{*}{$\begin{array}{c}\text { Total } \\
\text { Pacientes }\end{array}$} & \multicolumn{2}{|c|}{ Tipo de úlcera } & \multicolumn{2}{|c|}{ Sexo } & \multicolumn{3}{|c|}{ Ubicación } \\
\hline & & Aguda & Crónica & Hombres & Mujeres & $G$ & $\mathrm{D}$ & Yuit. \\
\hline \multicolumn{9}{|c|}{ Menos de } \\
\hline 1 & 3 & 3 & 0 & $\mathbf{I}$ & 2 & 1 & 1 & $1 *$ \\
\hline 1 a 2 & 3 & 2 & 1 & 3 & 0 & 1 & 1 & $1+$ \\
\hline 2 a 6 & 3 & 2 & $\mathbf{l}$ & 2 & $\mathbf{l}$ & 0 & 3 & 0 \\
\hline 6 a 10 & 11 & 0 & 11 & 6 & 5 & 0 & 11 & 0 \\
\hline \multicolumn{9}{|l|}{ más dc } \\
\hline 10 & 12 & 0 & 12 & 8 & 4 & 1 & 11 & 0 \\
\hline \multirow[t]{2}{*}{ TOTAL } & 32 & 7 & 25 & 20 & 12 & 3 & 27 & 2 \\
\hline & & $22 \%$ & $78 \%$ & $62 \%$ & $38 \%$ & $10 \%$ & $84 \%$ & $6 \%$ \\
\hline
\end{tabular}

$\mathbf{G}=$ Gástrica $; \mathbf{D}=$ Duodenał; * = Esótago, Estómago y Duadeno; $+=$ Estómago y Duodeno. 
te fue más frecuente que la hemorragia digestiva. (Tabla 2).

Tabla 2.

Marufestaciones clínicas en pacientes con úlceras agudas y erónicas.

\begin{tabular}{lcc}
\hline $\begin{array}{l}\text { Manifestaciones } \\
\text { clinicas }\end{array}$ & $\begin{array}{c}\text { Tipo de úlceras } \\
\text { Aguda } \\
\mathbf{n}=7\end{array}$ & $\begin{array}{c}\text { Crónica } \\
\mathbf{n}=25\end{array}$ \\
\hline $\begin{array}{l}\text { Hematemesis y melena } \\
\text { Hematernesis }\end{array}$ & 1 & 6 \\
$\begin{array}{l}\text { Melena } \\
\text { Dolor típico } \\
\text { (con ritmo horario) }\end{array}$ & 5 & 2 \\
$\begin{array}{l}\text { Dolor atipico } \\
\text { (sin ritmo horario) }\end{array}$ & - & 17 \\
$\begin{array}{l}\text { Dolor nocturno } \\
\text { Vómito crónico }\end{array}$ & - & 4 \\
Abdomen agudo & - & 11 \\
(perforación) & - & 11 \\
\hline
\end{tabular}

El dolor nocturno y los vómitos recurrentes fueron notables en esté grupo. Sólo en un enfermo la perforación duodenal señaló el comienzo de las manifestaciones clínicas. El motivo de la primera consulta de los enfermos con úlcera crónica fue dolor abdominal en $12(48 \%)$, hemorragia digestiva en $9(36 \%)$, vómito recurrente en $3(12 \%)$ y perforación en uno $(4 \%)$.

La acidez gástrica fue estudiada en 11 pacien. tes con úlcera crónica ( 10 duodenales y l piló. rico). Los resultados, expresados en $\mathrm{mEq} x \mathrm{~kg} x$ hora, aparecen en la Figura 1. El débito basal $(0.148 \pm 0.003)$ como el débito máximo $(0,417 \pm 0.006)$ fueron significativamente superiores a los respectivos valores del grupo control (débito basal $0,05 \pm 0,01$ y débito máximo $0,183 \pm 0,03$ ) (Fig. 1).

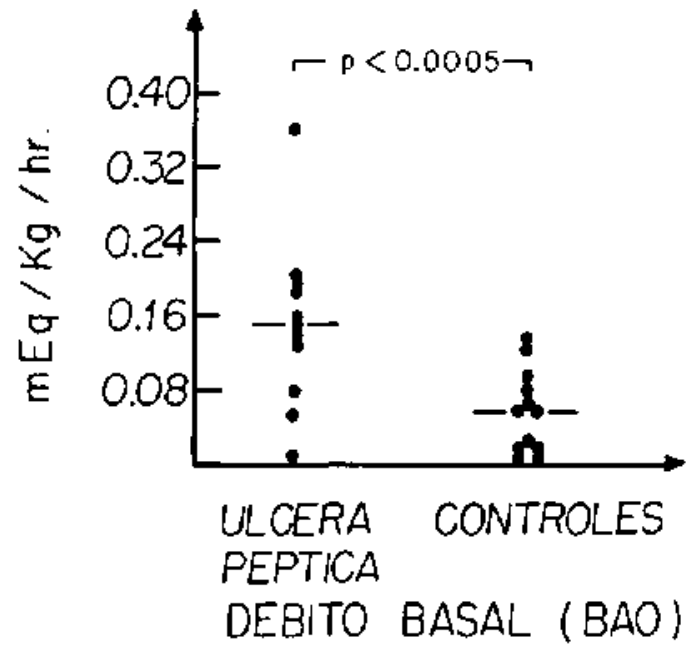

De los 32 pacientes, 27 recibieron tratamiento exclusivamente médico que consistió en reposos inicial, restricción de estimulantes de secreción gástrica (bebidas gaseosas, café, condimentos, etc.), antiácidos (hidróxido de $\mathrm{Mg}$ y Al), y cimetidina. Esta última droga fue empleada en 11 casos de úlcera crónica y en 6 de úlcera aguda. Todos los pacientes que ingresaron con hemorra. gia digestiva, requirieron algunas medidas adicionales como sonda nasogástrica, infusión de agua helada, transfusiones de sangre, etc.

Cinco pacientes ( 4 con úlcera crónica y I con úleera aguda) requirieron además tratamiento quirúrgico, to que representa $15 \%$ del total de pacientes de nuestra serie. Dos intervenciones sc hicicron con urgencia, por perforación duodenal, $y$ tres electivas, ana por hemorragias masivas recurrentes $y$ dos por sindrome de retención gástrica crónica.

De los 7 enfermos con úlceras agudás, 6 evolucionason satisfactoriamente y despues de un promedio de 10 meses de seguimiento no han repetido el episodio. En el nño con enfermedad poliquistica hepato-renal se desarrolló insuficiencià renal progresiva falleciendo 17 meses después de la hemorragia Gigestiva. El paciente con úlcera aguda operado por perforación sigue en buenas condiciones 16 meses después de la intervención.

De los 25 enfermos con úlcera crónica. 19 tuvieron una sola crisis ( $76 \%$ ) siendo seguidos en prome dio por 3 años. Seis desertaron sin completar el periodo minimo de tratamiento $y$ sin endoscopia de control. Diez. aún se encuentran en control, están asintomáticos y su endoscopia de control es normal. Se is enfermos con úlcera crónica sufrieron recurrencias de la enfermedad,

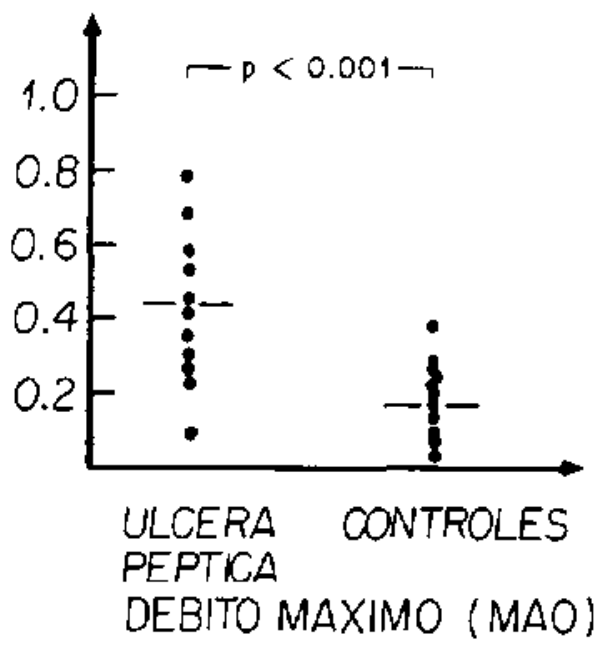

Figura 1. Producciön basal (BAO) y máxima (MAO de ácido en niños con úlcera péptica y controles. 
con evidencia clínica y endoscópica: uno, al cabo de cuatro episodios de hemorragia, fue sometido a vagotomía supraselectiva, después de lo cual sufrió un nuevo sangramiento; actualmente está asintomático, habiendo completado tres años desde la operación y un total de cinco afros de seguimiento. Otro niño que comenzó su enfermedad a los 2 años de edad fue operado por perforación duodenal a la vesicula y al pancreas. efectuándose vagotomia supraselectiva y gastrec. tomia tipo Billroth II; 5 años después sufrió una nueva hemorragia digestiva encontrándose en la endoscopia una úlcera en la boca anastomótica. Dos niños tuvieron varios episodios de dolor abdominal, presentando, recurrencia de la úlcera pre-pilórica que los llevó a un sindrome de retención gástrica. Por tal motivo fueron operados (Piloroplastia) después de lo cual cvolucionaron satisfactoriamente. Otros 2 niños tuvieron recurrencias clínicas y endoscópicas de la cnfermedad, uno sufrió sólo dolor abdominal, el otro dolor y hemorragia digestiva. En ambos se reinició el tratamiento médico y evolucionaron bien durante 4 años. Posteriormente fueron transferidos a un servicio de adultos por su cdad.

\section{DISCUSION}

En nuestra experiencia el empleo de la endoscopía digestiva alta significó un notable avance en la identificación de la úlcera péptica. Los pacientes descritos constituyen un grupo seleccionado en que la hemorragia digestiva alta y el dolor abdominal característico fueron las manifestaciones relevantes que motivaron su estudio. En estas condiciones no es posible obtcner informaeión más exacta sobre láa verdadera prevalencia de la úlcera péptica en rin̄os, lo que sólo sería posible con un protocolo prospectivo de mayor cobertura. Las estadisticas disponibles per. miten suponer que la úlcera péptica en la infancia ocurre con una frecuencia de 3,4 a 4,5 por 10.000 pacientes hospitalizados, siendo el sexo masculino el más afectado ${ }^{3}-4,8-9$.

El carácter restrospectivo de esta experienciá, sin un protocolo uniforme para elaborar el diagnóstico, tampoco permite conclusiones categóricas sobre la eficiencja comparativa de la endoscopía digestiva y los exámenes radiológicos. Diversas publicaciones han mostrado que la radiología identifica entre $25 \%$ y $50 \%$ de las úlceras gástricas y entre $50 \%$ y $89 \%$ de las úlceras duodenales confirmadas con endoscopia ${ }^{10-11}$. Las diferencias son mayores en las lesiones super. ficiales, difícilmente detectables con estudios baritados convencionales.
En el mismo período de este estudio hemos visto algunos casos con radiografías suge rentes de úlcera peptica en quienes la investigación endoscópica simultánea no confirmó las lesiones, hallazgo que debe ser tomado en cuenta por la distorsión que podria provocar diferentes casuís. ticas clinicas.

La fibroscopía digestiva constituye ил valioso elemento diagnóstico que puede ser utilizado desdc el período de recién nacido ${ }^{12}$. En una publicación anterior ${ }^{7}$, hemos destacado sus indicaciones y ventajas. En los casos de hemorragis digestiva alta este procedimiento debe ser utilizado precoz y prioritariamente para buscar la etiologia del sangramiento. Ante la sospecha de perforación gastrointestinal su empleo está contraindicado. Constituye un valioso complemento a la radiologia cuanto ésta es normal o dudosa $y$ hay fuertes elementos clinicos de sospecha de lcsión péptica. Ambos exámenes son complenentarios y no excluyentes; la radiología suela aportar una valiosa gama de información en rclación no sólo a lesiones estructurales sino que de la dinámica de los órganos investigados.

En la patogenia de la úlcera péptica intervie. nen varios factores. Situaciones tales como hipoxia, isquemia $y$ septicemia son capaces de provocar, aparentemente, úlccras agudas (de tensión), alterando la barrera mucosa que normalmente impide la difusión retrógrada de ionés hidrúgeno ${ }^{13}$. Los njôos de menor edad son par. ticularmente vulnerables a los factores atudidos, hecho en que nuestros resultados coinciden con lo descrito en la literatura ${ }^{3-4,14-15}$. La mayoria de nuestros pacientes con úlceras agudas tenian una coartación aórtica y en todos, la hemorragia digestiva apareció en el periodo postoperatorio inmediato, Hasta donde sabemos, en otras casuísticas sobre úlcera péptica infantil ésta no ha sido descrita en operados de coartación de aorta. Podría suponerse la existencia de una isquemia gastroduodenal crónica, que acentuada durante la operación podría influir en la génesis de la úlcera. Como no se hacen sistemáticamente endoscopias en todos los pacientes sometidos a reparación quirúrgica de la coartación, no nos es posible conocer con certeza la verdadera incidencia de lesiones gastroduodenales en estos pacientes, pero la hemorragia digestiva macroscópica, que es su manifestación principal, ocurrió en $5,6 \%$ de los operados.

Asimismo, sólo la investigación endoscópica sistemática de pacientes de unidades de tratamiento intensivo, quemados y recién nacidos críticamente enfermos, permitiría conocer la frecuencia real de úlceras pépticas en los njños 
con enfermedades graves. Es razonable suponer que esta complicación es más frecuente que lo aparente por sus síntomas (hemorragia, perforación) en este grupo de pacientes.

En los estudios epidemiológicos de úlcera péptica crónica frecuentemente se describe una historia familiar positiva, especialmente en los casos de úlcera duodenal. En nuestra experiencia, $74 \%$ de los pacientes con úlcera crónica, en quienes se conocia este antecedente, tenian familiares cercanos con la misma enfermedad. Este hecho es conocido y algunos autores publican proporciones similares de coincidencia ${ }^{16}$ aunque en otros ésta es inferior ${ }^{3-10-17}$. Los factores genéticos juegan un papel importante en la etiología de la úlcera, como ha quedado establecido en estudios realizados en gemelos.

El papel de la acidez gástrica también debe ser tomado en cuenta. Como los adultos, los niños con úlcera péptica tienden a presentar valores de acidez gástrica superiores a los controles ${ }^{1}$. Nuestros pacjentes tenían hiperacidez basal y postestímulo, lo que difiere parcialmente con los. resultados de Danús $s^{5}$ que encontró hiperacidez basal sin aumento de la máxima, $y$ de la experiencia de $\mathrm{C}_{\text {ristie y }}$ Ament ${ }^{19}$ cuyos pacientes mostraron la situación opuesta.

La úlcera crónica es más frecuente que la aguda en nuestra serie de enfermos y en otras ${ }^{3}$. 10 aunque algunos la tescriben en igual proporción ${ }^{4}$. El sexo masculino es el principal afecta$\mathrm{do}^{3-4-8}$. En esta y en la mayoría de las series publicadas, predominan las úlceras duodenales crónicas sobre las gástricas ${ }^{3-4-8}$. Sólo Nord y $\operatorname{cols}^{10}$ describicron lo contrario.

Las úlceras agudas parecen ubicarse con igual trecuencia en el estómado y el duodeno ${ }^{3-4-15}$ aunque nosotros, como Nuss y cols ${ }^{\mathbf{3}}$ encontramos un predominio de localizaciones duodenales.

El tratamicito que empleamos en nuestros pacientes se respalda en experiencias similares en niños ${ }^{5-6}$ pero taitan estudios controlados de doble ciego que validen el uso de cimetidina o antiácidos a esta edad. a diferencia de lo que ocurre en el adulto

Eł tratamicnto quirúrgico está indicado sn las hemorragias incontrolables, perforación y abstrucción ${ }^{2}$. La evolución de los pacientes operados no difieren de experiencias en adultos con úlcera péptica y sugierc que la evolución en niños está sujeta las nismas contingencias que en los adultos.

Las recurrencias en pacientes pediätricos acurren en 20 a $78 \%$ de los casos en los seguimientos prolongados $2-3-20-21$ y en numerosos casos los síntomas persisten en la edad adulta. Por esta razón el control de estos pacientes por tiempo prolongado es importante para conocer la incidencia de las complicaciones, y el momento óptimo para realizar la cirugía electiva ${ }^{20-23}$

\section{RESUMEN}

Se describe una revisión retrospectiva de 32 casos de úlcera péptica en los últimos 10 años del hospital Luis Calvo Mackenna. Treinta y uno confirmados por endoscopia o estudio radiológi. co o ambos $y$ uno durante una intervención quirúrgica por perforación duodenal. Desde que se comenzó a utilizar fibroscopia digestiva en 1978. se produjo un aumento significativo del número de casos diagnosticados. Veinticinco pacientes sufrian úlceras crónicas y siete úlceras agudas. Las crónicas se presentaron en 23 pacientes mayores de 6 años y 2 en menores de esta cdad. Todas las agudas ocurrieron en menores de 6 años y con mayor frecuencia durante el post operatorio inmediato de la reparación de una coartación de la aorta. Ambos tipos de lesiones se encontraron cun mayor frecuencia en el duodeno que en el estómago, y a fectan al sexo masculino más que al femenino en una proporción de 1,8:1. En $74 \%$ de los pacientes con úlcerd crónica habian familiares directos con antecedentes de la misma enfermedad. Todos los pacientes con úlcera aguda presentaron hemorragia digestiva al conienzo de la enfermedad, en cambio los pa. cientes con úlcera crónica presentaron dolor abdominal recurrente con mayor frecuencia que hemorragia digestiva. Los estudios de acidez gástrica en 11 pacientes con ílcera crónica mostraron valores de débito basal y máximo más clevado que los encontrados en 12 niños con dolor abdominal recurrente sin úlcera comprobada. De 25 casos con úlcera crónica, 19 tuvieron sólo una crisis $(76 \%)$ y 6 tuvieron dos o más (24\%). Recibiero tratamiento médico exclusivo 21 pacientes y 4 debieron ser operados ( $16 \%$ ).

\section{REFERENCIAS}

I. Ament, M.E.: Peptic ulcer disease in pediatric patients. En: "Digestive disease in children" Lebenthal E. ed. N.Y. Grune \& Stratton 1978, Pag. 421.

2. Nord, K., Lebenthat, $E$ : Peptic ulcer in children. A Ieview. Amer. J. Gastrónterol. 73: 75, 1980.

3. Deckelbaum, R., Roy, C. et al.: Peptic ulcer disease: a clinical study in 73 children. Can. Med. Assoc. Journal 111: 225, 1974 .

4. Tolia, V., Dubois, $R$.: Peptic Uice т disease in children an adolescents. A ten year experience. Clin. Pediat. 22: 665, 1983.

5. Donus, O.: Llcera péptica. En: "Problemas frecuentes en Gastroenterologia". Editorial Andrés Bello, Santiago, 1980 , pag. 45. 
6. Christie, D., Ament, M.: Diagnosis and treatment of duodenal ulcer in infancy and childhood. Pediatuic Ann. 5: 672, 1976

7. Wenger, I: Fibroscopía digestiva alta en niños. Experiencia de 204 procedimientos. Rev, Chì. Pediatr. 53: 411, 1982.

8. Nuss, $D$, Lynn, H.: Peptic ulceration in childhood. Surg. Clin. North Am. 51: 945, 1971.

9. Roy, C.C., Silverman, A., Cozzetto, F.: "Disorders of stomach and duodenum. En: "Textbook of pediatric clinical gastroenterology" 2a, ed. St. Louis. C.V. Mosby 1975: 155.

10. Nord, K., Rossi, $T$. et al.: Peptic ulcer in children. The predominance of gastric ulcers. Am. J. Gastroent. 75: 153, 1981.

11. Ament, M.E., Christie, D.L.: Uppet gastrointestinal fiberoptic endoscopy in pediatric patients. Gastroenterol. 72: 1244, 1977.

12. Leibman, W.M., Thaler, M.W., et al.: Endoscopic evaluation of upper gastrointestinal bleeding in newborn. Am. J. Gastroenterol. 69: 607, 1978.

13. Ysenberg. J., Richardson, Ch. et al.: Pathogenesis of peptic ulcer, En: "Gastrointestinal disease". Sleisenger, M. \& Fordtran, J Editores 2a. ed. Filadelíia. W.B. Saunders Co., 1978. Capitulo 46. Pag. 792.
14. Johnson, D., LHeureux, P. et al: Peptic ulcer disease in early infancy: clinical presentation and roentgenografic features. Acta Pediatr. Scand. 69: 753,1980 .

15. Dunn, St., Weber, T. et al.: Acute peptic ulcer in childhood. Emergency' surgical the rapy in 39 cases. Arch. Surg. 118: 656, 1983

16. Puri, $P$. Boyd, E.: Childten with duodenal ulcers and their families. Arch. Dis. Child. 50: 485, 1975.

17. Tudor, $R$.: Carta al editor. Lancet $2: 219,1974$.

18. Euler, A.R., Byrne, W.J. et ol..' Basal and pentagastrin-stimulated gastric acid secretory rates in normal children and in those with peptic ulcer disease. J. of Pediatr. 103: 776, 1983.

19. Christie, D.L., Ament, M.E.: Gastric acid hypersecretion in children with duodenal ulcer. Gastroenterol. ?1: 242, 1976 .

20. Puri, P., Boyd, E. et al.: Duodenal ulces in childhood: A continuing disease in adult life. $J$. Pediatt. Surg. 13: 525, 1978

21. Leix, F. Greaney, E.M.: Surgical experience with peptic ulce $x$ in infancy and childhood. Am. J. Surg. 106: 173,1963

22. Mohamed R., Mackay, C.: Peptic ulceration in adolescence. Br. J. Surg. 69: 525, 1982.

23. Maudar, K.K., Dutta, J. et al: Duodenal ulcer disease in children. World J. Surg. 4: 261, 1980. 